\title{
RhoA is dispensable for axon guidance of sensory neurons in the mouse dorsal root ganglia
}

\author{
Jennifer R. Leslie' ${ }^{1}$, Fumiyasu Imai ${ }^{1}$, Xuan Zhou ${ }^{2}$, Richard A. Lang ${ }^{1,3}$, Yi Zheng ${ }^{2}$ and Yutaka Yoshida ${ }^{1 *}$ \\ Divisions of Developmental Biology, Cincinnati Children's Hospital Medical Center, Cincinnati, OH, USA \\ ${ }^{2}$ Experimental Hematology and Cancer Biology, Cincinnati Children's Hospital Medical Center, Cincinnati, OH, USA \\ ${ }^{3}$ Pediatric Ophthalmology, Cincinnati Children's Hospital Medical Center, Cincinnati, OH, USA
}

\section{Edited by:}

James Jontes, Ohio State

University, USA

\section{Reviewed by:}

Zaven Kaprielian, Albert Einstein

College of Medicine of Yeshiva

University, USA

Tracy S. Tran, Rutgers University, USA

\section{${ }^{*}$ Correspondence:}

Yutaka Yoshida, Division of Developmental Biology, Cincinnati Children's Hospital Medical Center 3333 Burnet Avenue, Cincinnati, $\mathrm{OH} 45229$, USA.

e-mail: yutaka.yoshida@cchmc.org
RhoA, a member of the Rho family small GTPases, has been shown to play important roles in axon guidance. However, to date, the physiological function of RhoA in axon guidance events in vivo has not been determined genetically in animals. Here we show that RhoA mRNA is strongly expressed by sensory neurons in the developing mouse dorsal root ganglia (DRG). We have deleted RhoA in sensory neurons of the DRG using RhoA-floxed mice under the Wnt1-Cre driver in which Cre is strongly expressed in sensory neurons. Peripheral projections of sensory neurons appear normal and there are no detectable defects in the central projections of either cutaneous or proprioceptive sensory neurons in $R h o A^{f / f}$; Wht1-Cre mice. Furthermore, a co-culture assay using DRG explants from RhoA ${ }^{f / f}$; Wnt1-Cre embryos, and 293T cells expressing semaphorin3A (Sema3A) reveals that RhoA is not required for Sema3A-mediated axonal repulsion of sensory neurons. Expression of RhoC, a closely related family member, is increased in RhoA-deficient sensory neurons and may play a compensatory role in this context. Taken together, these genetic studies demonstrate that RhoA is dispensable for peripheral and central projections of sensory neurons in the DRG.

Keywords: RhoA, axon guidance, semaphorin, dorsal root ganglia, cutaneous sensory neurons, proprioceptive sensory neurons, spinal cord

\section{INTRODUCTION}

RhoA, a member of the small Rho GTPase family that regulates the cytoskeleton, has been implicated in various processes during the nervous system development, including the formation of adherens junctions, neuronal migration, and axon guidance (Giniger, 2002; Guan and Rao, 2003; Gallo and Letourneau, 2004; Heasman and Ridley, 2008; Hall and Lalli, 2010). The functions of RhoA in the mammalian nervous system have mainly been discerned from studies using a dominant negative or a knockdown approach. The physiological roles and functions of RhoA in the mammalian nervous system have just begun to be elucidated by loss-of-function studies using conditional genetargeting strategies (Herzog et al., 2011; Katayama et al., 2011; Cappello et al., 2012). These recent studies demonstrate that RhoA is essential for proper formation of adherens junctions and proliferation of neural progenitor cells in the mouse nervous system (Herzog et al., 2011; Katayama et al., 2011; Cappello et al., 2012), which is consistent with previous in vitro and invertebrate studies (Fukata and Kaibuchi, 2001; Bloor and Kiehart, 2002; Magie et al., 2002; Yamada and Nelson, 2007). However, it remained unclear whether RhoA is required for other functions, including axon guidance, in the mammalian nervous system.

Extensive studies using in vitro culture experiments have shown that activation of RhoA induces growth cone collapse and axonal repulsion by increasing actomyosin contractility (Giniger, 2002; Guan and Rao, 2003; Gallo and Letourneau,
2004; Heasman and Ridley, 2008; Hall and Lalli, 2010). For example, in vitro experiments have implicated RhoA in Sema3Amediated growth cone collapse of sensory neurons in the DRG (Dontchev and Letourneau, 2002; Wu et al., 2005; Hengst et al., 2006). Suppression of ROCK, a RhoA effector, by pharmacological inhibitors reduces Sema3A-induced growth cone collapse (Dontchev and Letourneau, 2002). In addition, Sema3A induces local translation of RhoA, and a knockdown approach reveals that RhoA is necessary for Sema3A-mediated growth cone collapse of DRG sensory neurons (Wu et al., 2005; Hengst et al., 2006). Despite these previous studies, the requirement of RhoA in Sema3A-dependent or -independent axonal repulsion in vivo during mammalian nervous system development remains unanswered.

To determine the physiological roles of RhoA in axon guidance, we have taken a loss-of-function approach. Since RhoA is strongly expressed by DRG neurons during development, RhoA was deleted from the DRG using RhoA-floxed mice together with Wnt1-Cre or Advillin-Cre mice in which Cre is expressed in the DRG. Surprisingly, loss of RhoA does not cause any obvious defects in the peripheral or central projections of DRG sensory neurons. In addition, RhoA is not required for Sema3A-mediated DRG axonal repulsion. Importantly, the protein level of RhoC, a related family member, is up-regulated in DRGs from $R h o A^{f / f}$; Wnt1-Cre embryos. Taken together, these findings suggest that RhoA itself is not essential for axon guidance of DRG sensory 
neurons and that RhoC may compensate for RhoA function in the DRG in vivo.

\section{MATERIALS AND METHODS MICE}

The following mouse strains were used in this study: RhoAfloxed (Chauhan et al., 2011; Katayama et al., 2011; Melendez et al., 2011), Wnt1-Cre (Danielian et al., 1998), and Advillin-Cre (da Silva et al., 2011). We used RhoA $A^{f / w}$; Wnt1-Cre or $R h o A^{f / w}$; Advillin-Cre mice as controls.

\section{TISSUE PREPARATION}

Spinal cords and their surrounding tissues were dissected from embryos at embryonic day (E) 10.5, E13.5, E15.5, E16.5, E17.5, and postnatal day (P) 1. They were then fixed with $4 \%$ paraformaldehyde (PFA) on ice for $2 \mathrm{~h}$ for immunofluorescence staining or overnight for in situ hybridization. Afterwards, they were cryoprotected in $30 \%$ sucrose, embedded in OCT compound, and sectioned at $16 \mu \mathrm{m}$.

\section{IMMUNOFLUORESCENCE}

For immunofluorescence, cryosections were stained with the following antibodies: rabbit anti-parvalbumine (PV) (Swant), rabbit anti-TrkA (R\&D systems), goat anti-TrkC (R\&D systems), rabbit anti-CGRP (Peninsula Lab), and guinea pig anti-vGlut1 (Chemicon). Alexa 488 and Cy3-conjugated secondary antibodies were purchased from Invitrogen and Jackson Immuno Research. Immunohistochemistry was performed as described (Leslie et al., 2011). Images were obtained using a LSM510 confocal microscope (Zeiss).

\section{In situ HYBRIDIZATION}

Digoxigenin (DIG)-labeled cRNA probes were used for in situ hybridization as described Schaeren-Wiemers and Gerfin-Moser (1993).

\section{DRG REPULSION ASSAY}

DRG explants from E12.5 embryos were co-cultured with 293T cell aggregates expressing Sema3A and/or GFP in collagen gel matrices for $48 \mathrm{~h}$ in the presence of NGF, then fixed with $4 \%$ PFA, and immunostained with mouse anti-Tuj1 antibody (Covance), and Cy3-conjugated secondary antibody. Images were obtained on an Axioplan microscope (Zeiss).

\section{WHOLE-MOUNT IMMUNOSTAINING}

Whole-mount immunostaining was performed on E10.5 embryos for neurofilament staining or E13.5 embyos for peripherin staining according the method described by Huber et al. (2005) and Mandai et al. (2009), respectively. Briefly, the embryos were fixed with 4\% PFA overnight, after which they were rinsed with phosphate buffered saline (PBS) three times and then fixed in Dent's fix (20\% DMSO, 80\% Methanol) overnight. The embryos were washed in PBS three times. The primary antibody, anti-neurofilament 2H3 (Developmental Studies Hybridoma Bank) or rabbit anti-peripherin (Millipore) was added in blocking solution (5\% normal goat serum, 75\% PBS, 20\% DMSO) and incubated at room temperature for 3-4 days. The embryos were washed with PBS five times for $1 \mathrm{~h}$ each. Then, an Alexa 488-conjugated secondary antibody (Invitrogen) was added in blocking solution and kept in the dark for 1-2 days. The embryos were washed five times in PBS before being viewed.

\section{IMMUNOBLOTTING}

Samples were lysed using RIPA buffer (Cell Signaling Technology) according to manufactory's suggestion. Supernatants were collected for SDS-PAGE and transferred to PVDF membrane (BioRad Laboratories). Specific protein expression was detected using the following antibodies: anti-RhoA (Cell Signaling Technology), anti-RhoC (Cell Signaling Technology), anti-Cdc42 (Cell Signaling Technology), anti-Lamin B (Santa cruz), and anti-Rac1 (BD Transduction Laboratories).

\section{RESULTS}

\section{RhOA IS EXPRESSED BY BOTH SENSORY AND MOTOR NEURONS}

To examine the expression pattern of RhoA, we performed in situ hybridization at various time points during development in the lumbar spinal cord and the DRG of wild-type mice. At E10.5, RhoA appears to be ubiquitously expressed throughout the spinal cord but showed relatively high expression in motor neurons and neural progenitors (Figure 1A). RhoA was expressed by most or all DRG sensory neurons at E10.5 (Figure 1E). At E13.5, strong expression of RhoA was detected in motor neurons (Figure 1B). At E16.5 and P1, similar to E13.5, RhoA was ubiquitously expressed in the spinal cord with high expression in motor neurons (Figures 1C,D). In the DRG, strong expression of RhoA was detected throughout development from E10.5 to P1 (Figures 1E-H). These expression analyses suggest that RhoA may have a role in spinal neurons including motor neurons and DRG sensory neurons. In this study, we focus on the expression of RhoA in the DRG.

\section{NO OBVIOUS DEFECTS IN PERIPHERAL PROJECTIONS OF SENSORY NEURONS IN $R h o A^{f / f}$; Wnt1-Cre EMBRYOS}

To determine the physiological roles of RhoA in DRG sensory neurons, we deleted RhoA in DRG sensory neurons using RhoA-floxed mice (Chauhan et al., 2011; Katayama et al., 2011; Melendez et al., 2011) together with Wnt1-Cre mice (Danielian et al., 1998; Hsu et al., 2010). Cre is expressed in the DRG and in the dorsal spinal cord of Wnt1-Cre mice (Danielian et al., 1998; Hsu et al., 2010). We confirmed that most RhoA expression was indeed deleted from DRG sensory neurons by performing Western blot analysis on DRG tissues from E12.5 control and Rho $A^{f / f}$; Wnt1-Cre embryos (Figure 2). As shown in Figure 2, RhoA was greatly reduced in RhoAf/f ; Wnt1-Cre embryos compared to control embryos. The expression of Cdc42 as well as Racl in the DRG was not changed in $R h o A^{f / f}$; Wnt1-Cre embryos compared to control embryos (Figure 2).

We first examined the peripheral projections of DRG sensory neurons of E10.5 Rho $A^{f / f}$; Wnt1-Cre embryos. To do this, we performed whole-mount immunostaining using anti-neurofilament antibody, which visualizes peripheral axonal projections of both sensory and motor neurons. DRG sensory neurons projected axons to the peripheral tissues of E10.5 RhoAf/f; Wnt1-Cre 


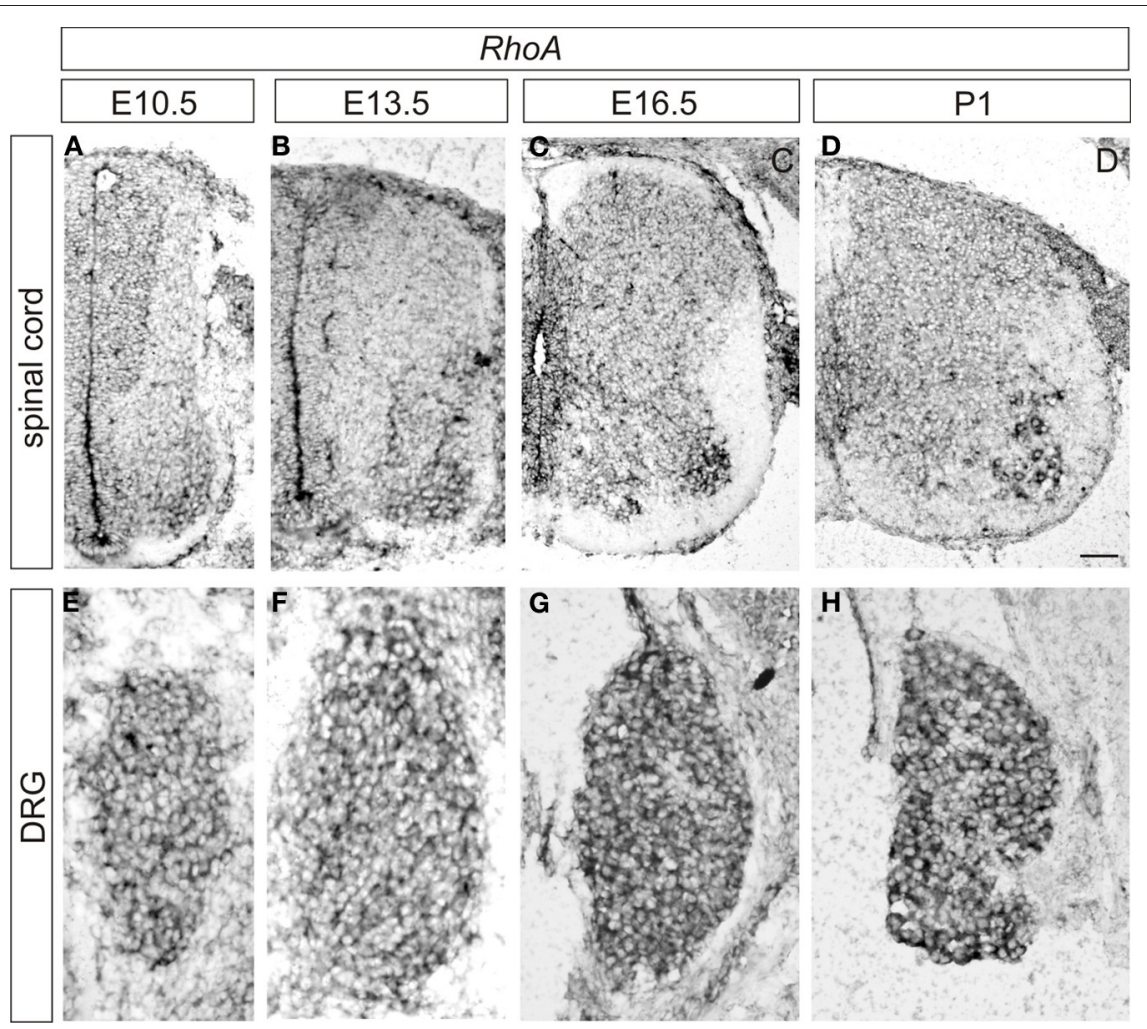

FIGURE 1 | Expression of $\boldsymbol{R h o A}$ in the developing mouse DRG and spinal cord. (A-D) Expression of RhoA in the spinal cord at E10.5 (A), E13.5 (B), E16.5 (C), and P1 (D). (E-H) Expression of RhoA in the DRG at E10.5 (A), E13.5 (B), E16.5 (C), and P1 (D). Scale bar, $50 \mu \mathrm{m}$.

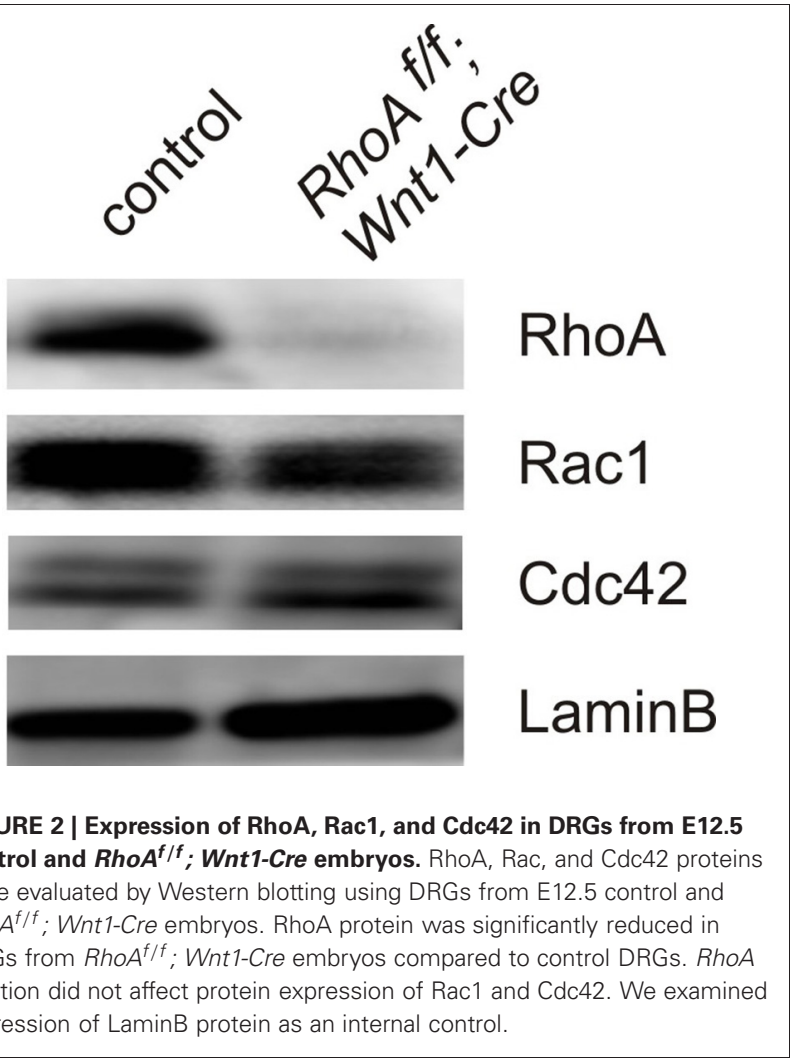

embryos similar to control embryos (Figures 3A-D). To further examine peripheral projections at E13.5, we performed whole-mount anti-peripherin immunostaining to visualize the peripheral axons in the distal limb (Mandai et al., 2009). We did not find any obvious defects in peripherin ${ }^{+}$peripheral axons in the distal limb of E13.5 RhoAf/f; Wnt1-Cre embryos (Figures 3E,F). Although we cannot exclude the subtle defects in peripheral axons in $R h o A^{f / f}$; Wht1-Cre embryos, RhoA is unlikely to have a major role in peripheral projections of DRG sensory neurons.

\section{PROPRIOCEPTIVE AXONAL PROJECTIONS SHOW TYPICAL PATTERNING IN $R h o A^{f / f}$; Wnt 1 -Cre EMBRYOS}

Next, we examined central projections of DRG sensory neurons in the spinal cord. DRG sensory neurons are subdivided into two major groups, proprioceptive and cutaneous sensory neurons (Brown, 1981; Koerber and Mendell, 1992). Proprioceptive neurons convey information about the state of muscle contraction and limb position, whereas cutaneous neurons mediate a wide range of noxious and innocuous stimuli (Brown, 1981; Koerber and Mendell, 1992). Proprioceptive sensory afferents project to the intermediate or ventral spinal cord, while cutaneous sensory neurons project their axons to the superficial dorsal horn (Brown, 1981; Koerber and Mendell, 1992). We analyzed the numbers of proprioceptive sensory neurons and proprioceptive axonal projections using an anti-Pv antibody, which marks all proprioceptive sensory neurons (Honda, 1995; Arber et al., 2000), 


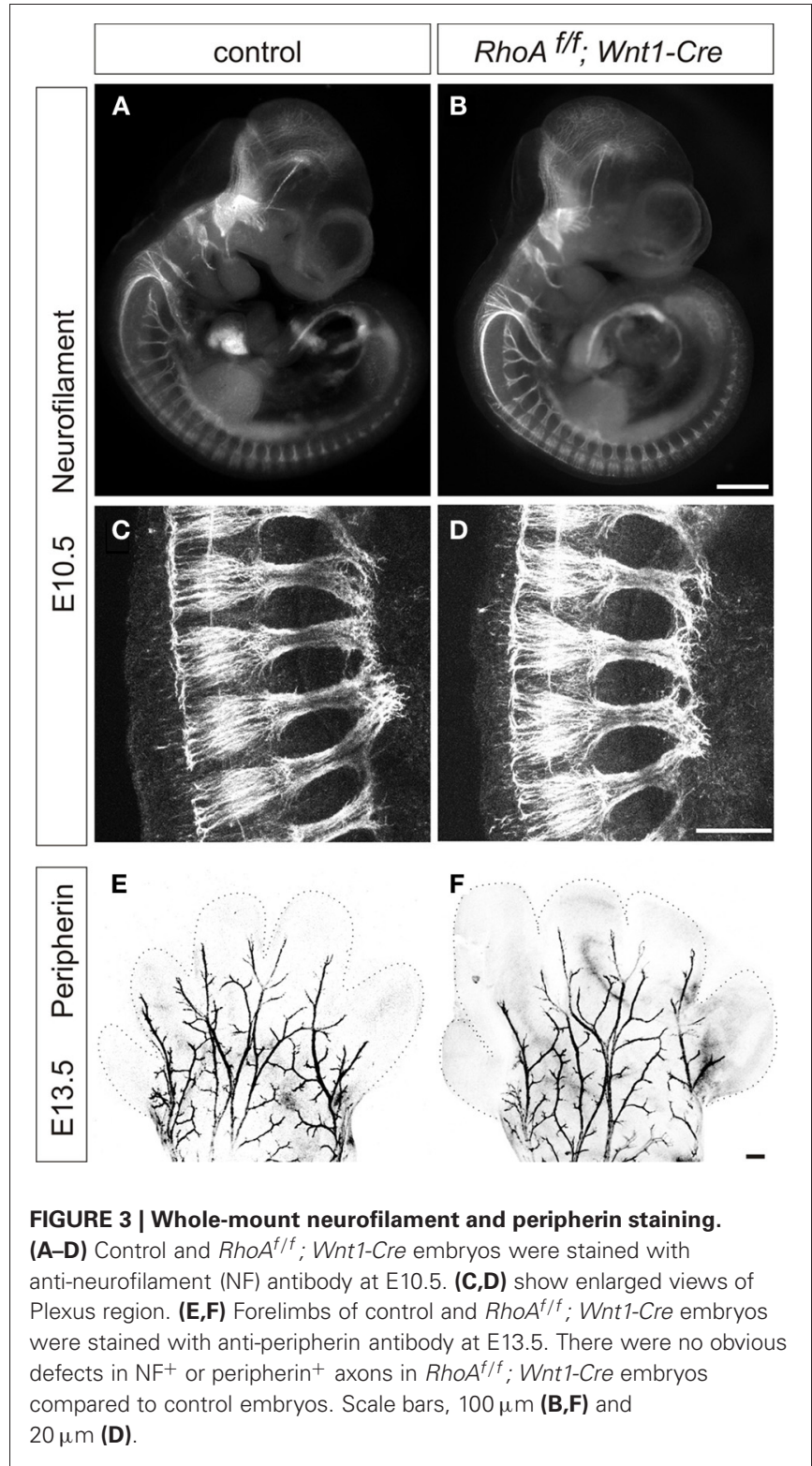

in $R h o A^{f / f}$; Wnt1-Cre embryos. There was no difference in the numbers of $\mathrm{Pv}^{+}$proprioceptive sensory neurons in the DRG between control and RhoA $A^{f f}$; Wnt1-Cre embryos at E15.5 and E17.5 (Figures 4A-D). In control embryos at E15.5 and E17.5, the proprioceptive axons entered the spinal cord medially and projected to the ventral spinal cord (Figures $4 \mathbf{E}, \mathbf{G}$ ). There were no obvious defects in proprioceptive axonal projections at E15.5 and E17.5 in RhoAf/f; Wnt1-Cre embryos compared to control embryos (Figures $\mathbf{4 E}-\mathbf{H}$ ). Thus, these data suggest that RhoA is not necessary for establishing proprioceptive axonal trajectories in the spinal cord.

\section{RhOA IS NOT INVOLVED IN ESTABLISHING CUTANEOUS AXONAL PROJECTIONS}

We next analyzed cutaneous sensory neurons in $R h o A^{f / f}$; Wnt1Cre embryos. To do this we performed immunohistochemistry

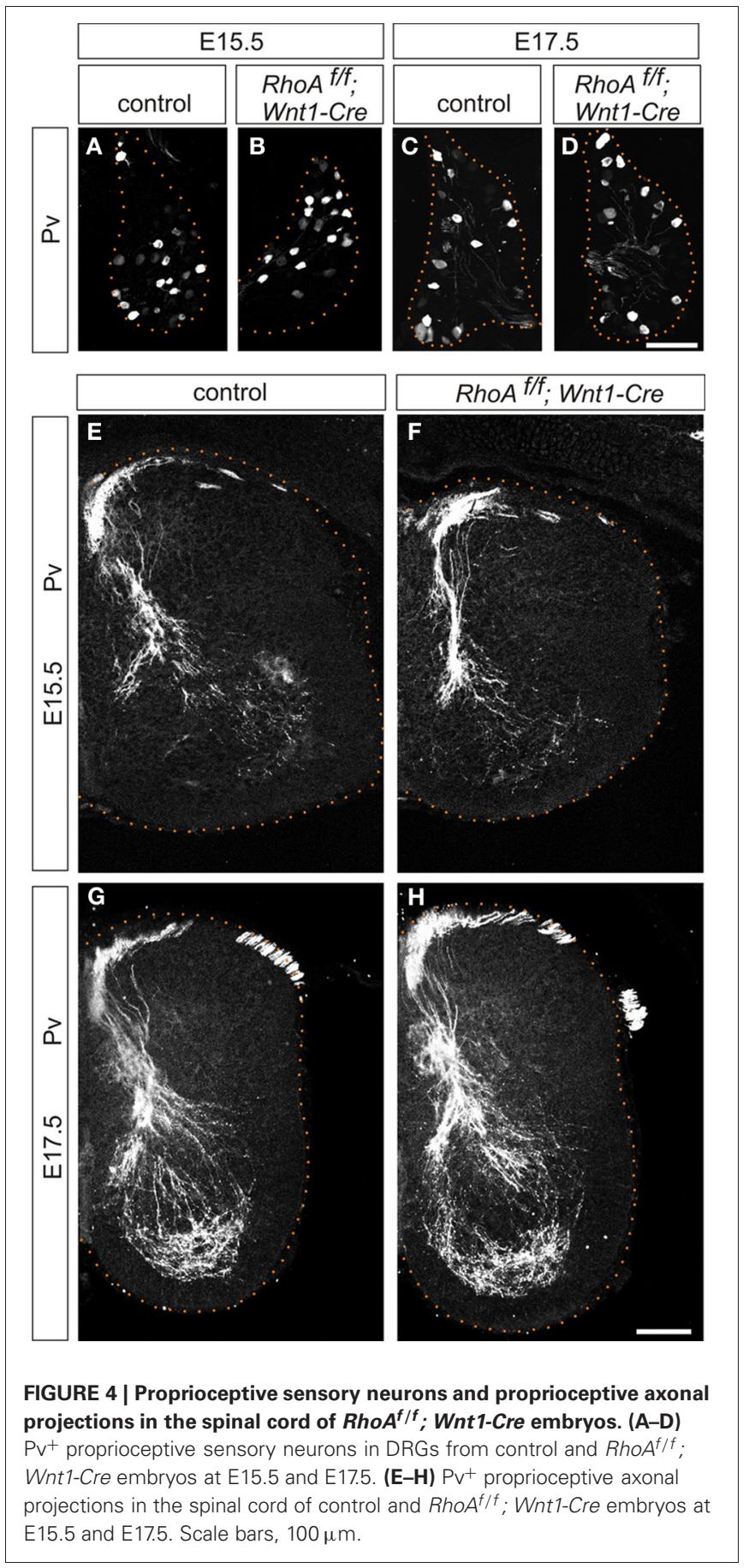

with an anti-TrkA antibody, which marks both cell bodies and axons of cutaneous sensory neurons during mouse embryogenesis. The numbers of $\operatorname{TrkA}^{+}$cutaneous sensory neurons in the DRG were not changed between E13.5-E17.5 control and $R h o A^{f / f}$; Wnt1-Cre embryos (Figures 5A,C,E,G,I,K). We also analyzed cutaneous axonal projections in the spinal cord in $R h o A^{f / f}$; Wnt1-Cre embryos. Cutaneous axons did not penetrate the spinal cord at E13.5, but penetrated the spinal cord laterally and projected in the dorsal spinal cord at E15.5 and E17.5 


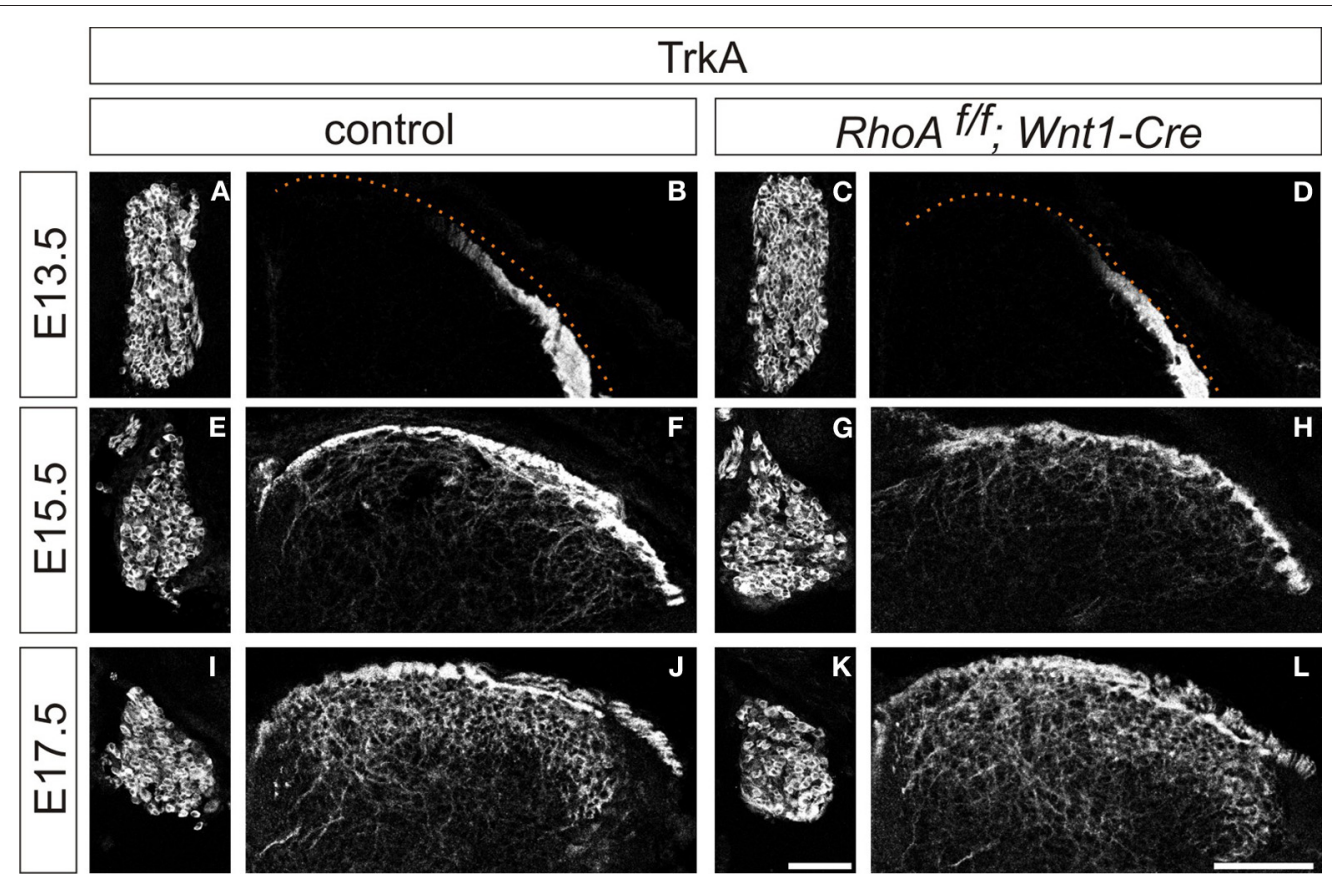

FIGURE 5 | Cutaneous sensory neurons and cutaneous axonal projections in the spinal cord of $R h o A^{f / f} ;$ Wnt1-Cre embryos. (A,E,I and $\mathbf{C}, \mathbf{G}, \mathbf{K}) \operatorname{TrkA}^{+}$cutaneous sensory neurons in DRGs from control and $R h o A^{f / f}$;
Wnt1-Cre embryos at E15.5 and E17.5. (B,F,J and $\mathbf{D}, \mathbf{H}, \mathbf{L})$ TrkA ${ }^{+}$cutaneous axonal projections in the spinal cord of control and $R$ ho $A^{f / f} ;$ Wnt1-Cre embryos at E15.5 and E17.5. Scale bars, $100 \mu \mathrm{m}$. in control embryos (Figures 5B,F,J). RhoA-deficient cutaneous axons displayed axonal trajectories similar to control embryos at E13.5, E15.5, and E17.5 (Figures 5D,H,L). Thus, RhoA is not necessary for the establishment of proper cutaneous axonal projections in the spinal cord at these embryonic stages. During postnatal development, cutaneous sensory neurons are further subdivided into different groups, which project axons to different laminae within the dorsal spinal cord. These different types of neurons are marked by different molecular markers. For example, calcitonin-gene-related-peptide (CGRP)-positive thinly myelinated cutaneous axons terminate in lamina I and outer lamina II of the dorsal horn in the spinal cord (Lawson, 2002). Isolectin IB4-, a marker of some primary afferent $\mathrm{C}$ fibers, positive and non-myelinated cutaneous axons, terminate in lamina II (Molliver et al., 1997; Fang et al., 2006). Furthermore, vesicular glutamate transporter 1 (vGlut1)-positive myelinated cutaneous afferents terminate in laminae III-V (Todd et al., 2003). Since most RhoA $A^{f f}$; Wnt1-Cre mice died before birth with severe brain defects (Katayama et al., 2011; data not shown), we used another Cre driver mouse line, Advillin-Cre (da Silva et al., 2011), whose expression starts at E12.5 in the DRG (Hasegawa et al., 2007). Rho $A^{f / f}$; Advillin-Cre mice were born in normal numbers and survived into adulthood. We examined $\mathrm{CGPR}^{+}, \mathrm{IB}^{+}$, and vGlut $^{+}$axonal projections of cutaneous sensory neurons in the P8spinal cord of RhoAf/f; Advillin-Cre mice. There was clear laminar segregation of different classes of cutaneous sensory axons both in control and Rho $A^{f / f}$; Advillin-Cre mice (Figure 6). Therefore, RhoA is unlikely to be involved in regulating laminar specific cutaneous axonal projections in the spinal cord during early mouse postnatal stages.

\section{RhoA IS NOT REQUIRED FOR Sema3A-MEDIATED AXONAL REPULSION}

Since it has been reported that RhoA is required for Sema3Amediated growth cone collapse of DRG sensory neurons using a knockdown approach (Wu et al., 2005; Hengst et al., 2006), we examined Sema3A-mediated axonal repulsion of DRG neurons from $R h o A^{f / f}$; Wnt1-Cre embryos. To do this we performed cocultures of E12.5 DRG explants from control or RhoA $A^{f / f}$; Wnt1Cre embryos with $293 \mathrm{~T}$ cells expressing GFP and/or Sema3A. E12.5 DRG axons from control embryos were repelled by Sema3A (Figure 7B). Similarly, RhoA-deficient DRG axons were also repelled by Sema3A (Figure 7D). This is in comparison to the unperturbed axonal growth of either set of DRGs in the presence of only GFP-transfected 293T cells (Figures 7A,C). These data suggest that RhoA itself is not essential for Sema3A-mediated DRG axonal repulsion.

\section{RhoC IS UP-REGULATED IN THE DRG IN THE ABSENCE OF RhoA}

Since RhoA has other related family members (Wennerberg and Der, 2004; Wheeler and Ridley, 2004), they may have a redundant function with RhoA in the DRG. Therefore, we examined the expression of RhoC by Western Blot analysis, since RhoC seems to be functionally the closest family member to RhoA (Wennerberg and Der, 2004; Wheeler and Ridley, 2004). We found that RhoC was significantly up-regulated in the DRG from $R h o A^{f / f}$; Wnt1Cre embryos compared to control embryos (Figure 8). These results suggest that RhoC may compensate for RhoA in the DRG.

\section{DISCUSSION}

In this study we show that the conditional deletion of RhoA in the DRG using either Wnt1-Cre or Advillin-Cre drivers does not have 

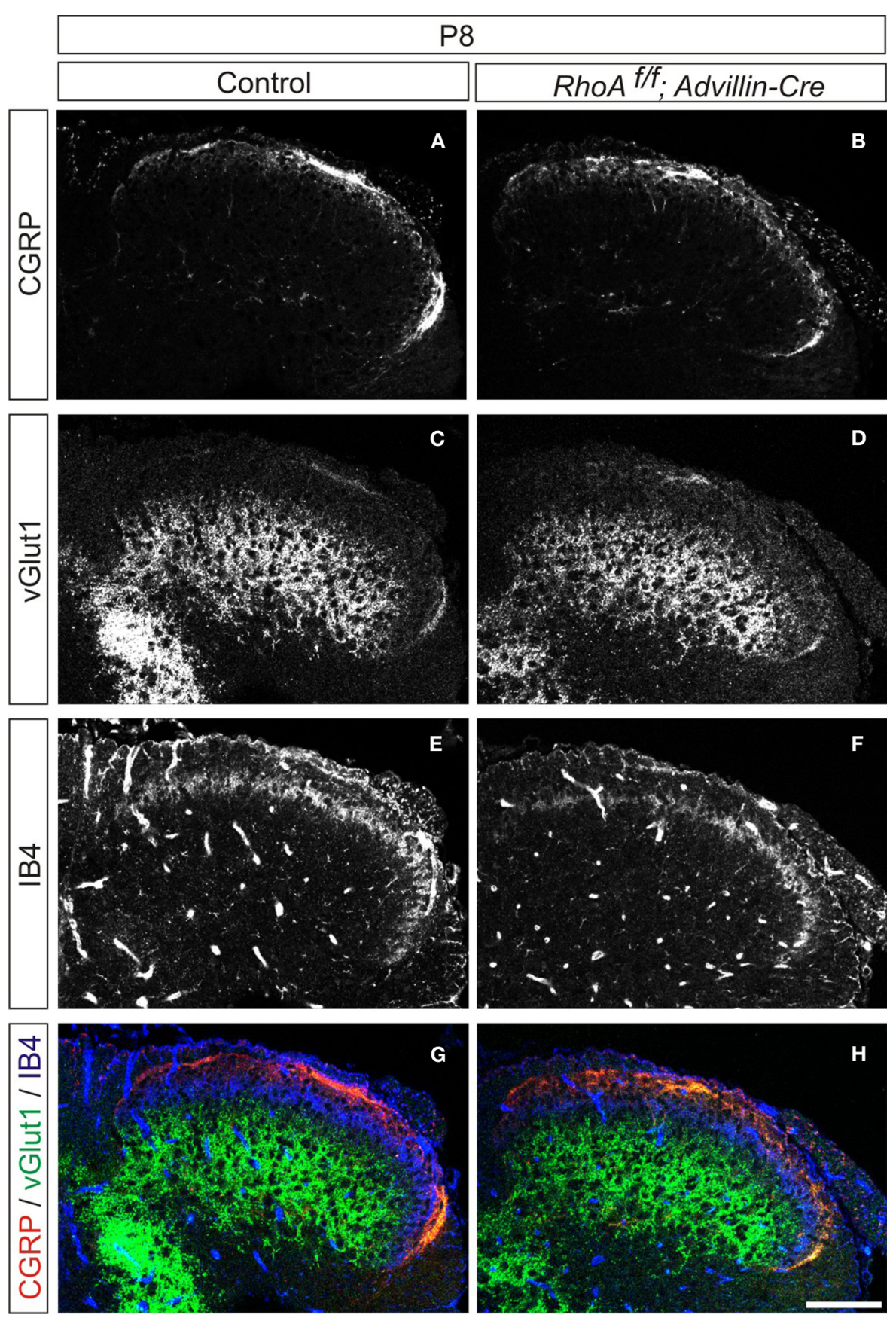

FIGURE 6 | Organization of cutaneous afferent projections in $\boldsymbol{R h o A}_{\boldsymbol{f}} \mathbf{f}$; Advillin-Cre mice. (A,B) CGRP expression, (C,D) vGlut1 expression,

(E,F) IB4-binding, and (G,H) merged views of CGRP (red), vGlut1 (green), and IB4-binding (blue) in P8 control and RhoA/f; Advillin-Cre mice. Scale bar, $100 \mu \mathrm{m}$

any obvious effect on either peripheral or central projections of DRG sensory neurons. In addition, loss of RhoA in the DRG does not change responses of DRG axons to Sema3A. Furthermore, RhoC protein is increased in the DRGs of RhoA $A^{f / f}$; Wnt1-Cre embryos compared to control embryos. This suggests that RhoA itself is not required for axon guidance of DRG sensory neurons and that the related protein RhoC may compensate for loss of RhoA function.

\section{LOSS OF RhOA DOES NOT CAUSE ANY OBVIOUS DEFECTS IN PROPRIOCEPTIVE OR CUTANEOUS AXONAL PROJECTIONS}

Many previous in vitro and in vivo studies have suggested important roles for RhoA in axon guidance (Giniger, 2002; Guan and Rao, 2003; Gallo and Letourneau, 2004; Heasman and Ridley, 2008; Hall and Lalli, 2010). However, it remained unknown whether RhoA is in fact required for axon guidance events in vivo in the mammalian nervous system, since a loss-of-function 

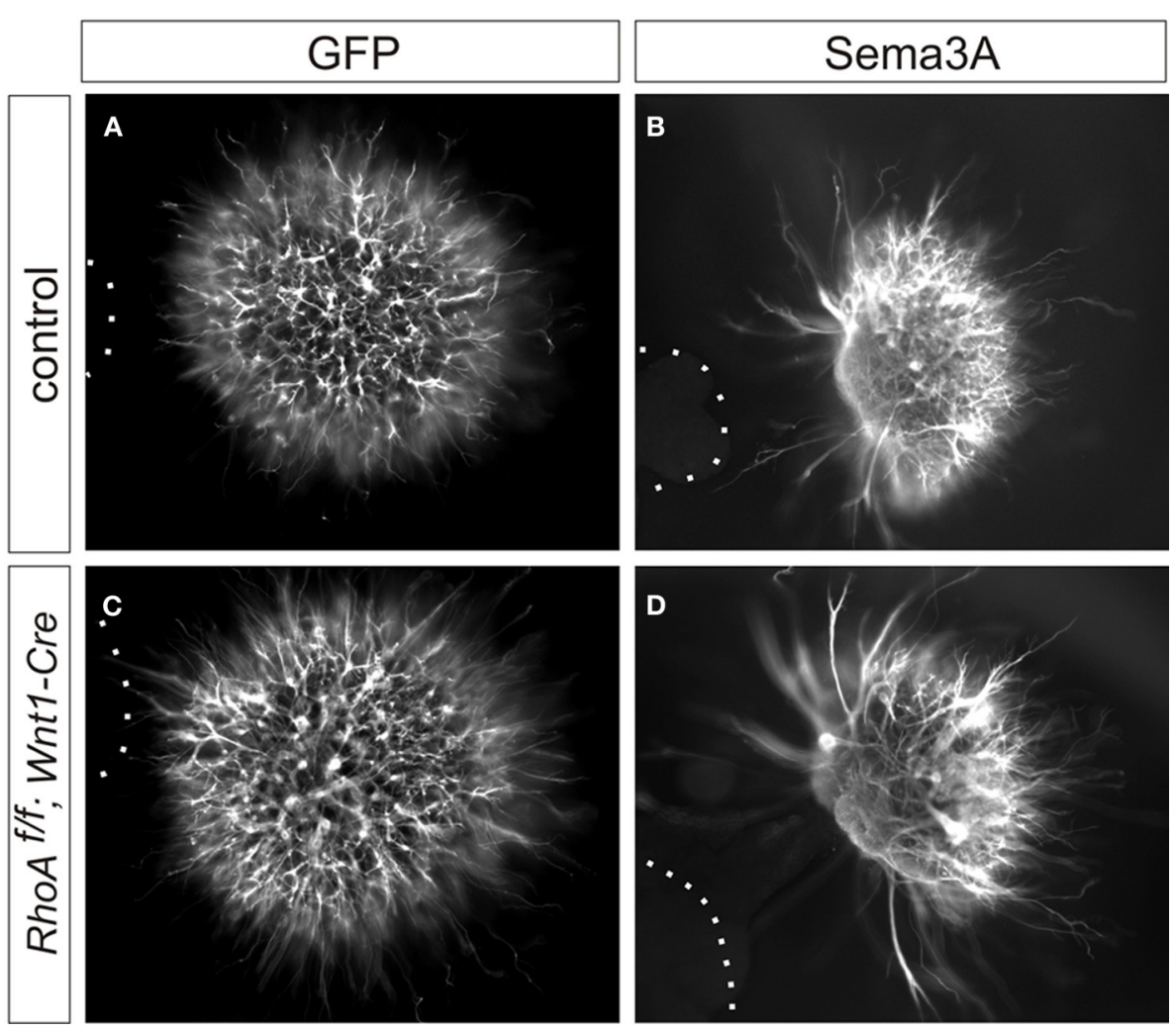

FIGURE 7 | Sema3A-induced DRG axonal repulsion in vitro. (A-D) Co-culture of DRG explants from control and RhoA $A^{f / f}$; Wht1-Cre embryos with GFP $(\mathbf{A}, \mathbf{C})$, or Sema3A and GFP $(\mathbf{B}, \mathbf{D})$ transfected cell aggregates. The axons of DRG neurons were examined by anti-TuJ1 antibody. Both DRG axons from control and RhoA ${ }^{f / f}$; Wnt 1-Cre embryos were repelled by Sema3A. Dotted lines outline aggregates of 293T cells.
A
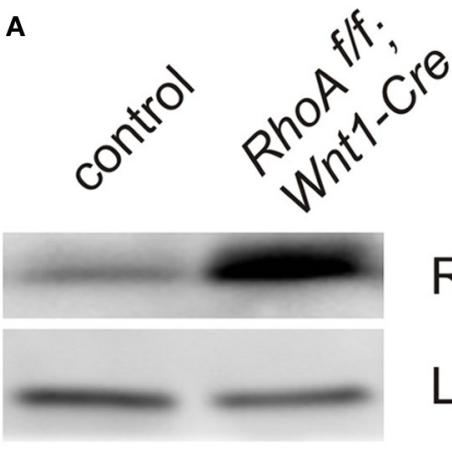

RhoC

\section{LaminB}

FIGURE 8 | Expression of RhoC in DRGs from E12.5 control and $R h o A^{f / f}$; Wnt1-Cre embryos. (A) RhoC protein was evaluated by Western blotting using DRGs from E12.5 control and RhoA $A^{f / f}$; Wht1-Cre embryos, and RhoC protein was up-regulated in DRGs from RhoAfff; Wht1-Cre embryos

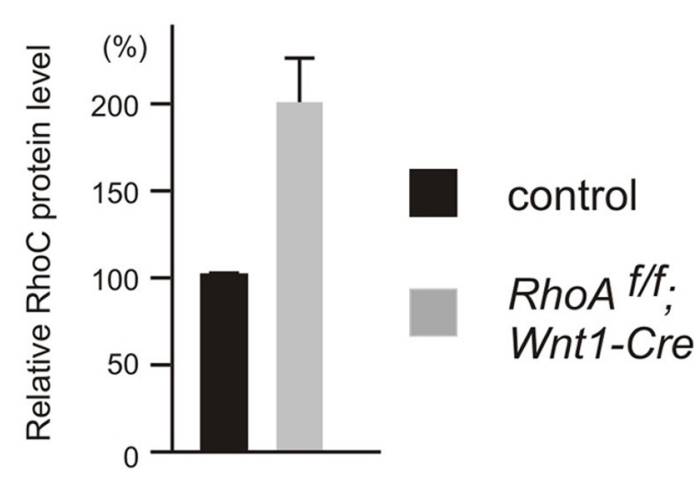

compared to control DRGs. (B) The quantification of levels of RhoC protein expression in DRGs from E12.5 control and RhoA f/f; Wnt1-Cre embryos. We determined expression of LaminB protein as an internal control $(n=4)$.

RhoA using Wnt1-Cre mice did not cause any aberrant peripheral axonal projections of sensory neurons that were detectable using anti-neurofilament and anti-peripherin antibodies. Second, there was no obvious disruption in the axon guidance of central projections of proprioceptive or cutaneous sensory axons. Therefore, it appears that RhoA itself is not crucial for the proper axonal pathfinding of DRG sensory neurons. 


\section{RhoA-DEFICIENT SENSORY AXONS STILL RESPOND TO Sema3A}

The small Rho GTPases have been shown to control axon guidance in part through semaphorin-plexin signaling (Kruger et al., 2005; Tran et al., 2007). For example, RhoA mRNA is localized to axons and growth cones of DRG sensory neurons, and this localization is mediated by an axonal targeting element located in the $3^{\prime}$ untranslated region of RhoA (Wu et al., 2005). Sema3A induces intra-axonal translation of RhoA mRNA, and this local RhoA translation has been suggested to be necessary for Sema3A-mediated growth cone collapse using a knockdown approach (Wu et al., 2005; Hengst et al., 2006). However, our loss-of-function study together with DRG explants cultured with Sema3A expressing 293T cells reveals that RhoA itself is not necessary for Sema3A-mediated axonal repulsion. The difference in the approach between loss-of-function and acute knockdown by siRNA or particular assay parameters to examine growth cone collapse or axonal repulsion may explain this discrepancy. Loss of Sema3A or its receptor neuropilin1 (Npn1) causes defects in fasciculation of peripheral motor and sensory axons (Behar et al., 1996; Kitsukawa et al., 1997; Taniguchi et al., 1997; Gu et al., 2003; Huettl et al., 2011). Our results showing no obvious defects in fasciculation of RhoA-deficient sensory axons (Figure 3) further support the idea that Sema3A-Npn1 signaling is present in RhoA $A^{f / f}$; Wnt1-Cre mice.

\section{RhOC EXPRESSION IS UP-REGULATED IN THE DRGs FROM $R h o A^{f / f} ;$ Wnt1-Cre EMBRYOS}

There are many different types of small Rho GTPases, and the Rho GTPases with the most similar structure to that of RhoA are RhoB and RhoC (Wennerberg and Der, 2004; Wheeler and Ridley, 2004). RhoA, RhoB, and RhoC are identical in approximately $85 \%$ of their protein sequence (Wennerberg and Der,

\section{REFERENCES}

Adamson, P., Paterson, H. F., and Hall, A. (1992). Intracellular localization of the P21rho proteins. J. Cell Biol. 119, 617-627.

Arber, S., Ladle, D. R., Lin, J. H., Frank, E., and Jessell, T. M. (2000). ETS gene Er81 controls the formation of functional connections between group Ia sensory afferents and motor neurons. Cell 101, 485-498.

Behar, O., Golden, J. A., Mashimo, H., Schoen, F. J., and Fishman, M. C. (1996). Semaphorin III is needed for normal patterning and growth of nerves, bones and heart. Nature 383, 525-528.

Bloor, J. W., and Kiehart, D. P. (2002). Drosophila RhoA regulates the cytoskeleton and cell-cell adhesion in the developing epidermis. Development 129, 3173-3183.

Brown, A. G. (1981). Organization in the Spinal Cord. New York, NY: Springer.

Cappello, S., Bohringer, C. R., Bergami, M., Conzelmann, K. K., Ghanem,
A., Tomassy, G. S., Arlotta, P., Mainardi, M., Allegra, M., Caleo, M., van Hengel, J., Brakebusch, C., and Gotz, M. (2012). A radial glia-specific role of RhoA in double cortex formation. Neuron 73, 911-924.

Chauhan, B. K., Lou, M., Zheng, Y., and Lang, R. A. (2011). Balanced Racl and RhoA activities regulate cell shape and drive invagination morphogenesis in epithelia. Proc. Natl. Acad. Sci. U.S.A. 108, 18289-18294.

Chen, Z., Sun, J., Pradines, A., Favre, G., Adnane, J., and Sebti, S. M. (2000). Both farnesylated and geranylgeranylated RhoB inhibit malignant transformation and suppress human tumor growth in nude mice. J. Biol. Chem. 275, 17974-17978.

Danielian, P. S., Muccino, D., Rowitch, D. H., Michael, S. K., and McMahon, A. P. (1998). Modification of gene activity in mouse embryos in utero by a tamoxifen-inducible form of

2004; Wheeler and Ridley, 2004), however, the localization of RhoB is different from that of RhoA and RhoC. RhoA and RhoC are both localized in the cytoplasms or at the plasma membrane, whereas RhoB is generally found in the endosomal membranes since RhoB has a unique $\mathrm{C}$-terminal lipid modifications and controls endosomal trafficking of membrane receptors (Adamson et al., 1992; Wheeler and Ridley, 2004; Heasman and Ridley, 2008). In addition, RhoB seems to have a growth inhibitory effect, whereas RhoA and RhoC have the opposite effect (Du and Prendergast, 1999; Chen et al., 2000; Wennerberg and Der, 2004). Thus, RhoB is likely to be functionally distinct from RhoA and RhoC. We also found that RhoC is significantly up-regulated in the DRG from RhoA f/f; Wnt1-Cre embryos. Therefore, RhoC might be able to compensate for RhoA in the DRG. The analysis of RhoA and $R h o C$ double mutants will give us valuable information about the possibility of the functional redundancy between RhoA and $\mathrm{RhoC}$ in the nervous system in the future.

In conclusion, our findings presented here using a loss-offunction approach demonstrate that RhoA itself is not essential for axonal projections of DRG sensory neurons and that RhoC may compensate for RhoA in the DRG. Further studies will reveal whether RhoA itself is required for the axon guidance of other types of neurons in the nervous system and whether RhoA and RhoC have redundant functions in the DRG and other regions of the mammalian nervous system.

\section{ACKNOWLEDGMENTS}

We thank F. Wang for providing Advillin-Cre mice, C. Y. Kuan for providing reagents and materials, and K. Campbell and D. R. Ladle for helpful comments on this manuscript. Yutaka Yoshida was supported by grants from NINDS (NS065048).

Cre recombinase. Curr. Biol. 8, 1323-1326.

da Silva, S., Hasegawa, H., Scott, A., Zhou, X., Wagner, A. K., Han, B. X., and Wang, F. (2011). Proper formation of whisker barrelettes requires periphery-derived Smad4 dependent TGF-beta signaling. Proc. Natl. Acad. Sci. U.S.A. 108 3395-3400.

Dontchev, V. D., and Letourneau, P. C. (2002). Nerve growth factor and semaphorin $3 \mathrm{~A}$ signaling pathways interact in regulating sensory neuronal growth cone motility. J. Neurosci. 22, 6659-6669.

Du, W., and Prendergast, G. C. (1999). Geranylgeranylated RhoB mediates suppression of human tumor cell growth by farnesyltransferase inhibitors. Cancer Res. 59, 5492-5496.

Fang, X., Djouhri, L., McMullan, S., Berry, C., Waxman, S. G., Okuse, K., and Lawson, S. N. (2006). Intense isolectin-B4 binding in rat dorsal root ganglion neurons distinguishes C-fiber nociceptors with broad action potentials and high Nav1.9 expression. J. Neurosci. 26, 7281-7292.

Fukata, M., and Kaibuchi, K. (2001). Rho-family GTPases in cadherin-mediated cell-cell adhesion. Nat. Rev. Mol. Cell Biol. 2, 887-897.

Gallo, G., and Letourneau, P. C. (2004). Regulation of growth cone actin filaments by guidance cues. $J$. Neurobiol. 58, 92-102.

Giniger, E. (2002). How do Rho family GTPases direct axon growth and guidance? A proposal relating signaling pathways to growth cone mechanics. Differentiation 385-396.

Gu, C., Rodriguez, E. R., Reimert, D. V., Shu, T., Fritzsch, B., Richards, L. J., Kolodkin, A. L., and Ginty, D. D. (2003). Neuropilin-1 conveys semaphorin and VEGF signaling during neural and cardiovascular development. Dev. Cell 5, 45-57.

Guan, K. L., and Rao, Y. (2003). Signalling mechanisms mediating neuronal responses to guidance cues. Nat. Rev. Neurosci. 4, 941-956. 
Hall, A., and Lalli, G. (2010). Rho and Ras GTPases in axon growth, guidance, and branching. Cold Spring Harb. Perspect. Biol. 2, a001818.

Hasegawa, H., Abbott, S., Han, B. X., Qi, Y., and Wang, F. (2007). Analyzing somatosensory axon projections with the sensory neuron-specific Advillin gene. J Neurosci. 27, 14404-14414.

Heasman, S. J., and Ridley, A. J. (2008). Mammalian Rho GTPases: new insights into their functions from in vivo studies. Nat. Rev. Mol. Cell Biol. 9, 690-701.

Hengst, U., Cox, L. J., Macosko, E. Z., and Jaffrey, S. R. (2006). Functional and selective RNA interference in developing axons and growth cones. J. Neurosci. 26, 5727-5732.

Herzog, D., Loetscher, P., van Hengel, J., Knüsel, S., Brakebusch, C., Taylor, V., Suter, U., and Relvas, J. B. (2011). The small GTPase RhoA is required to maintain spinal cord neuroepithelium organization and the neural stem cell pool. J. Neurosci. 31, 5120-5130.

Honda, C. N. (1995). Differential distribution of calbindin-D28k and parvalbumin in somatic and visceral sensory neurons. Neuroscience 68, 883-892.

Hsu, W., Mirando, A. J., and Yu, H. M. (2010). Manipulating gene activity in Wnt1-expressing precursors of neural epithelial and neural crest cells. Dev. Dyn. 239, 338-345.

Huber, A. B., Kania, A., Tran, T. S., Gu, C., De Marco Garcia, N., Lieberam, I., Johnson, D., Jessell, T. M., Ginty, D. D., and Kolodkin, A. L. (2005). Distinct roles for secreted semaphorin signaling in spinal motor axon guidance. Neuron 48, 949-964.

Huettl, R. E., Soellner, H., Bianchi, E., Novitch, B. G., and Huber, A. B. (2011). Npn-1 contributes to axon-axon interactions that differentially control sensory and motor innervation of the limb. PLoS Biol. 9:e1001020. doi: 10.1371/journal.pbio.1001020

Katayama, K., Melendez, J., Baumann, J. M., Leslie, J. R., Chauhan, B. K., Nemkul, N., Lang, R. A., Kuan, C. Y., Zheng, Y., and Yoshida, Y. (2011). Loss of RhoA in neural progenitor cells causes the disruption of adherens junctions and hyperproliferation. Proc. Natl. Acad. Sci. U.S.A. 108. 7607-7612.

Kitsukawa, T., Shimizu, M., Sanbo, M., Hirata, T., Taniguchi, M., Bekku, Y., Yagi, T., and Fujisawa, H. (1997). Neuropilin-semaphorin III/Dmediated chemorepulsive signals play a crucial role in peripheral nerve projection in mice. Neuron 19, 995-1005.

Koerber, H. R., and Mendell, L. M. (1992). "Functional heterogeneity of dorsal root ganglion cells," in Sensory Neurons: Diversity, Development and Plasticity, ed S. A. Scott (New York, NY: Oxford University Press), 77-96.

Kruger, R. P., Aurandt, J., and Guan, K. L. (2005). Semaphorins command cells to move. Nat. Rev. Mol. Cell Biol. 6, 789-800.

Lawson, S. N. (2002). Phenotype and function of somatic primary afferent nociceptive neurones with C-, Adelta- or Aalpha/beta-fibres. Exp. Physiol. 87, 239-244.

Leslie, J. R., Imai, F., Fukuhara, K., Takegahara, N., Rizvi, T. A., Friedel, R. H., Wang, F., Kumanogoh, A., and Yoshida, Y. (2011). Ectopic myelinating oligodendrocytes in the dorsal spinal cord as a consequence of altered semaphorin 6D signaling inhibit synapse formation. Development 138, 4085-4095.

Magie, C. R., Pinto-Santini, D., and Parkhurst, S. M. (2002). Rhol interacts with p120ctn and alphacatenin, and regulates cadherinbased adherens junction components in Drosophila. Development 129, 3771-3782.

Mandai, K., Guo, T., St Hillaire, C., Meabon, J. S., Kanning, K. C.,
Bothwell, M., and Ginty, D. D. (2009). LIG family receptor tyrosine kinase-associated proteins modulate growth factor signals during neural development. Neuron 63 614-627.

Melendez, J., Stengel, K., Zhou, X., Chauhan, B. K., Debidda, M., Andreassen, P., Lang, R. A., and Zheng, Y. (2011). RhoA GTPase is dispensable for actomyosin regulation but is essential for mitosis in primary mouse embryonic fibroblasts. J. Biol. Chem. 286 15132-15137.

Molliver, D. C., Wright, D. E., Leitner, M. L., Parsadanian, A. S., Doster, K. Wen, D., Yan, Q., and Snider, W. D. (1997). IB4-binding DRG neurons switch from NGF to GDNF dependence in early postnatal life. Neuron 19, 849-861.

Schaeren-Wiemers, N., and GerfinMoser, A. (1993). A single protocol to detect transcripts of various types and expression levels in neural tissue and cultured cells: in situ hybridization using digoxigenin-labelled cRNA probes. Histochemistry 100, 431-440.

Taniguchi, M., Yuasa, S., Fujisawa, H., Naruse, I., Saga, S., Mishina, M. and Yagi, T. (1997). Disruption of semaphorin III/D gene causes severe abnormality in peripheral nerve projection. Neuron 19, 519-530.

Todd, A. J., Hughes, D. I., Polgar, E., Nagy, G. G., Mackie, M., Ottersen, O. P., and Maxwell, D. J. (2003). The expression of vesicular glutamate transporters VGLUT1 and VGLUT2 in neurochemically defined axonal populations in the rat spinal cord with emphasis on the dorsal horn Eur. J. Neurosci. 17, 13-27.

Tran, T. S., Kolodkin, A. L., and Bharadwaj, R. (2007). Semaphorin regulation of cellular morphology. Annu. Rev. Cell Dev. Biol. 23, 263-292.

Wennerberg, K., and Der, C. J. (2004). Rho-family GTPases: it's not only
Rac and Rho (and I like it). J. Cell Sci. 117(Pt 8), 1301-1312.

Wheeler, A. P., and Ridley, A. J. (2004). Why three Rho proteins? RhoA, RhoB, RhoC, and cell motility. Exp. Cell Res. 301, 43-49.

Wu, K. Y., Hengst, U., Cox, L. J., Macosko, E. Z., Jeromin, A., Urquhart, E. R., and Jaffrey, S. R. (2005). Local translation of RhoA regulates growth cone collapse. Nature 436, 1020-1024.

Yamada, S., and Nelson, W. J. (2007). Localized zones of Rho and Rac activities drive initiation and expansion of epithelial cellcell adhesion. J. Cell Biol. 178, 517-527.

Yoshida, Y., Han, B., Mendelsohn, M., and Jessell, T. M. (2006). PlexinA1 signaling directs the segregation of proprioceptive sensory axons in the developing spinal cord. Neuron 52, 775-788.

Conflict of Interest Statement: The authors declare that the research was conducted in the absence of any commercial or financial relationships that could be construed as a potential conflict of interest.

Received: 29 February 2012; paper pending published: 19 March 2012; accepted: 09 May 2012; published online: 22 May 2012.

Citation: Leslie JR, Imai F, Zhou X, Lang RA, Zheng Y and Yoshida Y (2012) RhoA is dispensable for axon guidance of sensory neurons in the mouse dorsal root ganglia. Front. Mol. Neurosci. 5:67. doi: 10.3389/fnmol.2012.00067 Copyright (c) 2012 Leslie, Imai, Zhou, Lang, Zheng and Yoshida. This is an open-access article distributed under the terms of the Creative Commons Attribution Non Commercial License, which permits non-commercial use, distribution, and reproduction in other forums, provided the original authors and source are credited. 